\title{
Anisotropies in the Perception of Stereoscopic Surfaces: the Role of Orientation Disparity
}

\author{
RON CAGENELLO, BRIAN J. ROGERS $†$ \\ Received 19 August 1992; in revised form 24 March 1993
}

\begin{abstract}
We measured stereoscopic slant detection thresholds for surfaces slanting about a horizontal or a vertical axis. For random-dot covered surfaces, 1.25 deg of slant was required to detect slant about a horizontal axis, whereas $2.1 \mathrm{deg}$ of slant was required to detect slant about a vertical axis. This anisotropy could be due to the fact that orientation disparities, which contain information about surface slant, are generally smaller for surfaces slanting about a vertical axis. To test this possibility, slant thresholds were measured for surfaces whose orientation disparity content was manipulated independently of the other slant information present. When the magnitude of orientation disparity was the same for surfaces slanting about a horizontal and a vertical axis, both surface orientations required about 1.5 deg of slant to be detected; thus the anisotropy became negligible. In contrast, when the orientation disparity content of a surface slanting about a vertical axis was zero, $3-4$ deg of slant was required for detection; thus the anisotropy became larger. Under the conditions of these experiments, it appears that the visual system utilizes orientation disparities.
\end{abstract}

Stereopsis Binocular vision Stereoscopic slant Orientation disparity Anisotropy

\section{INTRODUCTION}

Stereoscopically-defined surfaces which slant or curve about a horizontal axis are often perceived more readily, and have more apparent slant or curvature, than surfaces which slant or curve about a vertical axis (Wallach \& Bacon, 1976; Rogers \& Graham, 1983; Gillam, Flagg \& Finlay, 1984; Gillam, Chambers \& Russo, 1988; Rogers \& Cagenello, 1989; Mitchison \& McKee, 1990; Mitchison \& Westheimer, 1990; Gillam \& Ryan, 1992). Although there are significant individual differences in the magnitude of the effect, studies of the anisotropy have shown that surfaces containing disparities that change in a direction orthogonal to the axis joining the two eyes [i.e. in a vertical direction with horizontallyoriented surfaces such as those illustrated in Fig. 1(a)] appear to have more depth than surfaces containing disparities that change in a direction parallel to the axis joining the two eyes [Fig. 1(b)].

This striking perceptual anisotropy is not limited to stereoscopic surfaces. Rogers and Graham (1983) have shown that there is an analogous effect in the perception of surfaces defined by motion parallax and used this fact to argue for a possible similarity in the mechanisms that extract the two different sources of information. Moreover, they showed that the anisotropy for perceiving

"Vision Research Laboratory, The Lighthouse Inc., 800 Second Avenue, New York, NY 10017, U.S.A.

$\nmid$ Department of Experimental Psychology, Oxford University, Oxford OX1 3UD, England. parallax surfaces is not a function of the spatial orientation of the surface per se (vertical or horizontal), but instead is a product of the spatial patterning of the velocity field. A surface slanting or curving about a horizontal axis (with depth changes in a vertical direction) generates a pattern of shearing motion with horizontal movements of the observer's head, while a surface slanting or curving about a vertical axis (with depth changes in a horizontal direction) generates an expansion-compression flow field (see Fig. 2).

If the anisotropy were due to the orientation (i.e. horizontal or vertical) of the surface per se, then horizontally-oriented surfaces ought to be easier to see and have more apparent depth than vertically-oriented surfaces whatever the underlying disparity transformation. Rogers and Graham reported instead that vertically-oriented surfaces were easier to see and had more apparent depth when the observer moved his or her head vertically. With vertical head movements, the two different flow field transformations are reversed for the two different surface orientations such that a vertically-oriented surface now generates a shearing flow field and a horizontally-oriented surface an expansioncompression flow field (Rogers \& Graham, op cit).

This result suggests that it is the expansioncompression pattern of relative motion that is responsible for the poorer depth-from-motion percept rather than the actual orientation of the three-dimensional surface. The equivalent experiment for stereoscopic surfaces is necessarily impossible, but Rogers and Graham 


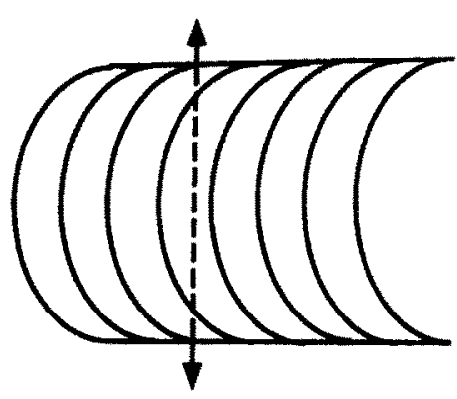

a





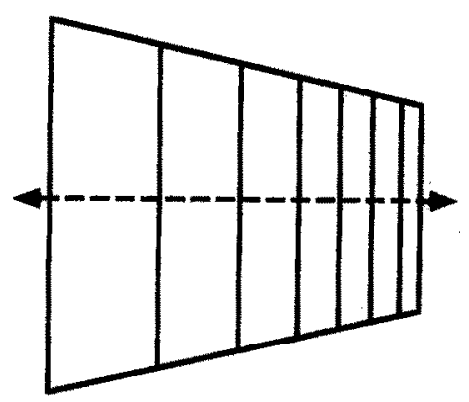

FIGURE 1. Examples of surfaces curving or slanting about a horizontal axis (a) or a vertical axis (b). The dashed arrows indicate the direction in which disparity is changing for these surfaces. (a) Disparities change in a direction orthogonal to the (horizontal) axis joining the two eyes. (b) Disparities change in a direction parallel to the axis joining the two eyes.

argued that it was more likely that the anisotropy in the perception of stereoscopic surfaces also results from the different spatial patterns of disparities generated by horizontally- and vertically-oriented surfaces.

Even if Rogers and Graham were correct in linking the anisotropy to the different spatial patterns of disparities generated by horizontal and vertical surfaces, this does not, by itself, provide a satisfactory explanation as to why expansion-compression patterns of disparities should be more difficult to detect than shearing patterns. In this paper we show that these two disparity transformations generate different magnitudes of orientation disparity, and that these differences can account for the reported anisotropy in the perception of stereoscopic surfaces at threshold.

Orientation disparity is defined as the difference in the two-dimensional orientation of corresponding elements in the two eyes' views (Blakemore, Fiorentini \& Maffei, 1972). It is a potentially useful source of information, because, for a given line element orientation (with respect to the cyclopean eye), the magnitude of orientation disparity is directly related to the magnitude of surface slant. Furthermore, orientation disparity could be calculated directly from corresponding retinal image features by binocular neurons with receptive fields tuned to a slightly different orientation in each eye. Both of these factors, the potential usefulness and the ease of computation, have motivated psychophysical, physiological, and computational investigations of orientation disparity (Mitchell \& O'Hagan, 1972; von der Heydt, 1978; von der Heydt, Hanny \& Dursteler, 1981; Ninio, 1985; DeValois, von der Heydt, Adorjani \& DeValois,
1975; Gillam \& Rogers, 1991; Blakemore et al., 1972; Nelson, Kato \& Bishop,1978; Koenderink \& van Doorn, 1976; Jones \& Malik, 1992; Wildes, 1991).

Figure 2 illustrates the effect of the direction of surface slant on orientation disparity. As indicated above, surfaces slanting about a horizontal axis [Fig. 2(a)] and viewed with horizontally separated eyes generate retinal images which are related to each other (to a first approximation) by a shearing transformation, as illustrated in the stereo pair. Surfaces slanting about a vertical axis [Fig. 2(b)] generate retinal images which are related to each other (to a first approximation) by an expansion in one image, and a compression in the other. In this paper, the terms shear and expansioncompression will be used subsequently as a shorthand to describe the disparity transformations generated by surfaces slanting about horizontal and vertical axes, respectively.

The difference in the orientation disparity content of the images in Fig. 2 can be seen immediately. In the expansion-compression case [Fig. 2(b)], there are no orientation differences between either corresponding horizontal or corresponding vertical lines in the binocular images, while in the shear case [Fig. 2(a)] there is a significant orientation disparity between corresponding vertical lines. The illustrated surfaces are, however, special cases in that they contain only horizontal and vertical lines. Figure 3 shows the complete functions relating arbitrary line orientations to orientation disparity for the shear and expansion-compression surfaces which have $1 \mathrm{deg}$ of slant with respect to the frontoparallel. Line orientation in this figure, and elsewhere in 

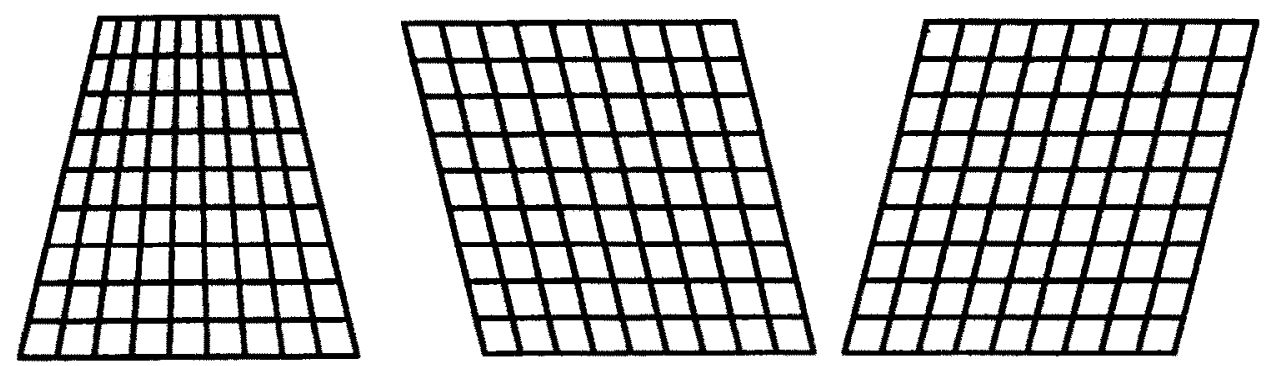

a
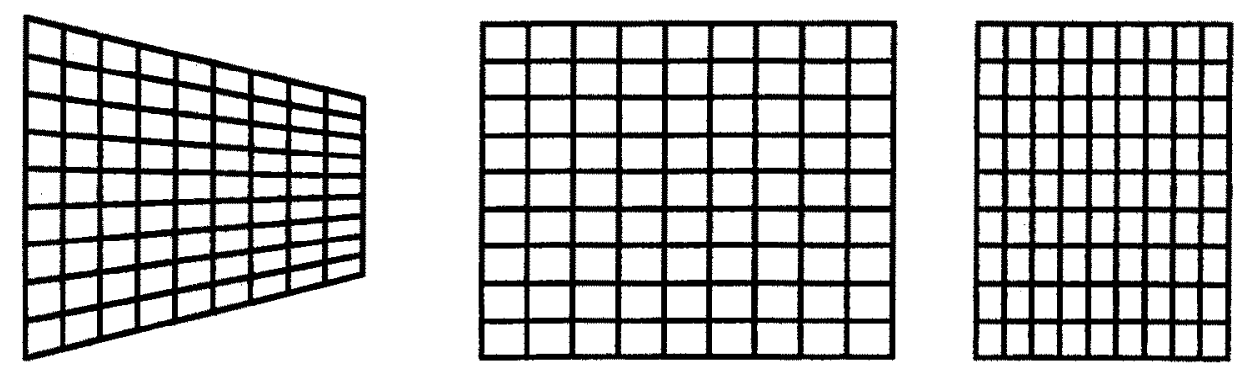

b

FIGURE 2. Surfaces ruled with vertical and horizontal lines slanting about a horizontal axis (a), or a vertical axis (b), and stereo pairs illustrating the disparity transformations. (a) The three-dimensional surface depicted on the left generates retinal images which can be approximated by a shearing transformation. Note the significant difference in orientation between corresponding vertical lines in the images. (b) The three-dimensional surface depicted on the left generates retinal images which can be approximated by an expansion in one image, and a compression in the other. Note that there is no difference in orientation for corresponding lines in the two images. The disparity transformations in this figure are exaggerated for illustrative

purposes, and are not intended to accurately represent the slant magnitude depicted by the perspective drawings.

the paper, refers either to orientation of a line in the image plane, prior to the application of the disparity transformation, or equivalently to orientation of a line in the plane of the slanting surface (i.e. orientation of lines ruling the surface), with $0 \mathrm{deg}$ being horizontal and 90 deg vertical.

The functions in Fig. 3 show how, for a given magnitude of surface slant, the magnitude of orientation disparity in stereoscopic images depends both on (i) the absolute (cyclopean) orientation of lines being viewed by the two eyes, and on (ii) the direction in which the surface is slanting. Specifically, the maximum orientation disparity generated by a surface slanting about a horizontal axis is generated by any vertical lines or markings on that surface. The orientation disparity between corresponding elements is zero for both vertical and horizontal lines ruling a surface which is slanting about a vertical axis [this can also be seen in Fig. 2(b)]. Lines oriented at $45 \mathrm{deg}$ generate the same magnitude of orientation disparity for both expansion-compression and shear surfaces. Finally, it can be seen that the maximum orientation disparity generated by a shear surface is twice as large as the maximum orientation disparity for an expansion-compression surface. Note that the degree of physical surface slant is the same for both surface types, and that neither the disparity gradients, nor the positional disparities present change when the orientation of lines covering a surface is changed. Hence, by varying the orientation of lines on a surface, orientation disparity can be manipulated independently of the other indicators of surface slant (Fig. 4).

Three major predictions concerning the detection of surface slant can be made on the basis of the functions shown in Fig. 3. First, if surface slant detection thresholds are determined solely by the maximum orientation disparity information present, thresholds for surfaces slanted about a vertical axis should be $100 \%$ higher than those for surfaces slanted about a horizontal axis. If, on the other hand, thresholds are determined by the average orientation disparity information present, thresholds for surfaces slanted about a vertical axis should be $57 \%$ higher. This figure was obtained by integrating the area under the functions shown in Fig. 3, and means that for a random (isotropic) arrangement of oriented features in the scene, the average magnitude of orientation disparities will be $57 \%$ larger for surfaces slanting about a horizontal axis. Second, the orientation disparity hypothesis predicts that similar detection thresholds (i.e. no anisotropy) should be found for the two surface types when they are ruled with $45 \mathrm{deg}$ line elements, because the orientation disparities generated in this case have the same magnitude (Fig. 3). Third, thresholds should be maximally different (i.e. the anisotropy should be largest) when the slanted surface is covered with vertical rulings, because the orientation disparities are zero for slants about a vertical axis, while the orientation disparities are maximal for slants about a horizontal axis. Note that the predictions are based on 


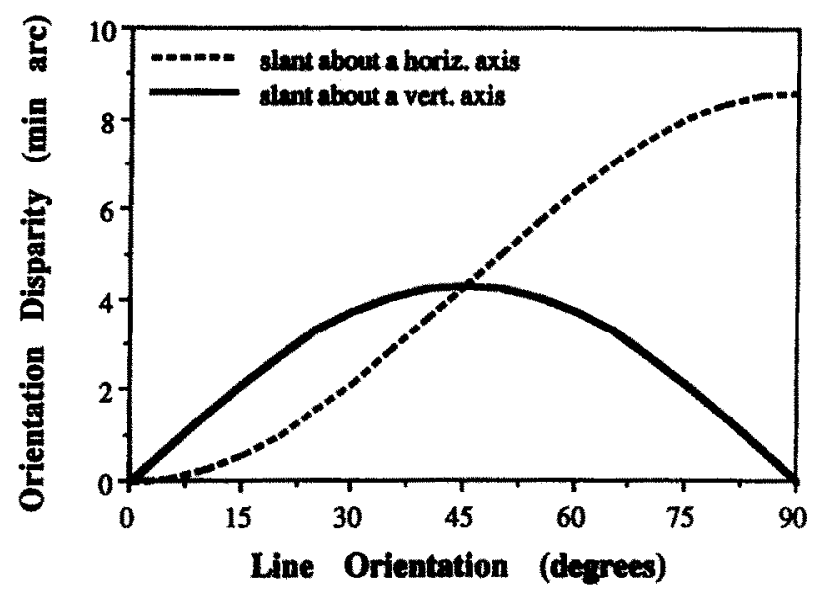

FIGURE 3. For a given surface slant (in this case, I deg from fronto-parallel), the orientation disparity of a binocularly corresponding line segment marking the surface depends on (i) the orientation of the line segment being viewed by the two eyes, and on (ii) the direction in which the surface is slanting. These functions relate line segment orientation ( $x$-axis) to orientation disparity $(y$-axis) for slant about a horizontal axis (dashed line) or slant about a vertical axis (solid line). A line orientation of $0 \mathrm{deg}$ is horizontal, $90 \mathrm{deg}$ is vertical. Note the following, as highlighted in the text: (1) the average orientation disparity (obtained by integrating the area under the curves) is $57 \%$ larger for a horizontally-oriented surface than for a vertically-oriented surface. (2) A vertical line generates the largest orientation disparity for a horizontally-oriented surface, but zero orientation disparity for a vertically-oriented surface. (3) The magnitude of orientation disparity generated by \pm 45 deg lines is the same for horizontally- and verticallyoriented surfaces.

the assumption that detection thresholds are determined by orientation disparities alone.

\section{EXPERIMENT ONE}

To test these predictions, detection thresholds for slanted surfaces were measured as a function of the orientation of lines covering the surfaces. The underlying rationale of this experiment is as follows. Under normal viewing conditions, both point disparities and orientation disparities will be generated by three-dimensional stereoscopic surfaces. In order to assess the influence of orientation disparity on task performance, positional disparities must either be removed as a consistent source of information, or kept constant while orientation disparities are manipulated independently. In his study of orientation disparity, von der Heydt (1978) employed the former technique by using uncorrelated dynamic noise stimuli. Thus, in the stimuli he used, there were no corresponding points to match in the two eyes' images but there was a consistent orientation difference between the (non-corresponding) line elements in the two eyes' images. Our stimuli, on the other hand, were designed so that orientation disparities could be independently manipulated while keeping positional disparities constant (and co-existing with orientation disparity as they normally would be). This was accomplished by varying the orientation of a grid of lines in the image plane prior to applying the appropriate disparity transformation (Fig. 4; see also Fig. 5). If the visual system is capable of using orientation disparities, we should expect detection thresholds to vary with the orientation of lines ruled on three-dimensional stereoscopic surfaces.

\section{Methods}

Experiments were run under the control of a Cromemco System Three computer equipped with a GPIB (general purpose interface bus) parallel interface and several serial ports. The computer controlled a Wavetek 175 Arbitrary Waveform Function Generator through the GPIB, and A/Ds, D/As and an experimental control box through the serial ports. A Matrox graphics board $(256 \times 256 \times 1$ bit resolution) generated the graphical output which was displayed on two Hewlett Packard 1304A large screen oscilloscopes (P31 phosphor). A custom-built PAL standard TV ramp signal generator converted the line $(\mathrm{X})$ and frame $(\mathrm{Y})$ synchronization pulses provided by the Matrox board into ramp signals to drive the $\mathrm{X}$ and $\mathrm{Y}$ scans of the oscilloscopes, in order to create a raster display. The brightness $(Z)$ output from the graphics board was fed directly into the $Z$ input of the scopes. The differential $X, Y$ and $Z$ inputs of the HP 1304 oscilloscopes allow additional signals to be added linearly to the line, frame, and intensity input signals. See Rogers and Graham (1982) for further details of this set-up.

Initially, the images presented to the two eyes were identical and yielded a percept of a single, fused flat surface lying in a fronto-parallel plane. To generate a pattern of horizontal disparities between the left and



FIGURE 4. Illustration of a square figure with a 45 deg line drawn through it before (solid line) and after (dashed line) a shear transformation. The two figures could be thought of as superimposed left and right halves of a stereo pair. $\Delta \theta_{90}$ and $\Delta \theta_{45}$ are the orientation disparities resulting from the shear transformation, for the vertical and 45 deg lines respectively. It can be seen that $\Delta \theta_{90}>\Delta \theta_{45}$, as depicted in Fig. 3. $\delta$ is the maximum horizontal disparity generated by the shear transformation, and it is the same for the line orientations $\theta_{90}$ and $\theta_{45}$ $S$ is the image's vertical size, the area and direction over which $\delta$ is changing. $S$ does not change under the shear transformation. The change in disparity over the image in a vertical direction, $\Delta \delta / S$, which is the disparity gradient, is the same for the two line orientations. Thus for a given shear transformation (this holds for the expansion-compression transformation as well), changing the line orientation only changes the orientation disparity, not the maximum positional disparity or the gradient of disparity in the vertical (horizon tal for expansion-compression) direction. 
right eye displays, ramp waveforms of equal and opposite amplitudes from the Wavetek function generator were fed into the additional $\mathrm{X}$ inputs of the two scopes. The analog disparity signal was thus completely independent of the digital graphics image. This technique allowed us to create continuously varying, equal and opposite disparities between the two eyes which were not limited by the pixel size of the displays. When fused by the two eyes, the disparate images yielded the percept of a solid surface which slanted smoothly in depth.

The oscilloscopes were placed in a modified Wheatstone configuration at a viewing distance of $57 \mathrm{~cm}$. To ensure that the vergence angle was appropriate for this viewing distance the mirrors closest to the scopes were adjusted to bring the fused image into alignment (in depth) with a rod placed $57 \mathrm{~cm}$ away. The outer two mirrors remained fixed at $45 \mathrm{deg}$. Prior to each data collection session, the gains of the display oscilloscopes were matched as closely as possible by calibrating them with a physical graticule pattern on a perspex sheet. Observers viewed the display in a darkened room with their heads held in place by a chinrest.

Slant detection thresholds were obtained using a forced choice procedure in which subjects indicated the slant direction of a planar surface patch with respect to the fronto-parallel. The displayed surface slanted either about a vertical axis (the expansion-compression condition) or about a horizontal axis (the shear condition). In the expansion-compression condition the slant of the surface appeared as either a "left wall" [left side closer, as in Fig. 2(b)] or as a "right wall" (right side closer), whereas in the shear condition the surface appeared as either a "ground plane" [bottom closer, as in Fig. 2(a)] or a "sky plane" (top closer).

Stimuli were composed of either (i) $50 \%$ random light/dark dots, or (ii) a grid composed of 0 and $90 \mathrm{deg}$ lines, or (iii) a grid composed of +45 and $-45 \mathrm{deg}$ lines. These were presented as circular patches subtending $10.66 \mathrm{deg}$ of visual angle. Figure 5 shows examples of the oriented grids prior to and after each disparity transformation. An individual bright dot subtended $2.5 \mathrm{~min}$ arc, and dot separation (dot center to dot center) was $5 \mathrm{~min}$ arc. Therefore, 0 and $90 \mathrm{deg}$ lines were made up of bright dots separated by $5 \mathrm{~min}$ arc while in the +45 and $-45 \mathrm{deg}$ lines the bright dot separation was $7 \mathrm{~min}$ $\operatorname{arc}(5 \times \sqrt{2})$. The lines in the $0 / 90$ deg grid were separated by $1.33 \mathrm{deg}$ horizontally and vertically, giving a total of eight lines. The $\pm 45 \mathrm{deg}$ grid was a rotated version of the $0 / 90 \mathrm{deg}$ grid, so the line separation was the same $(1.33 \mathrm{deg})$, though the minimum horizontal and vertical distance between line intersections was larger by a factor of $\sqrt{2}$.

In each experimental session, subjects made 70 observations, 10 at each of seven disparity gradients (frontoparallel, plus three positive and three negative) presented in a random sequence for a single stimulus marking type (random dots, $0 / 90 \mathrm{deg}$ grid, or $\pm 45 \mathrm{deg}$ grid) and disparity transformation type (shear or expansioncompression). Prior to the main experiment, practice sessions at each combination of the independent variables were undertaken to establish the appropriate range of slants for deriving a psychometric function, and to familiarize subjects with the procedure. A single trial proceeded as follows: a random-dot patch with zero disparity gradient (i.e. fronto-parallel) was presented for $1 \mathrm{sec}$, the screen was blanked for $1 \mathrm{sec}$, the stimulus was presented for $1.5 \mathrm{sec}$, and the screen was blanked until the observer made a response, at which time the cycle repeated until 70 observations had been made. The fronto-parallel random-dot patch between trials prevented the build-up of depth aftereffects and provided a reference surface.

Two experienced psychophysical observers with normal (BJR) and corrected-to-normal (RBC) eyesight took part, making a total of $350-420$ observations (50-60 per point on the psychometric function) for each pairing of stimulus marking type and disparity transformation type. A third experienced subject (SPM) took part in the oriented grid conditions only, making a total of 140 observations for each orientation and disparity transformation type.

\section{Results}

Best-fitting regression lines were estimated for each observer and each condition using probit analysis (Finney, 1971). Thresholds were taken as the inverse of the slope of each regression line, which corresponds to the $84 \%$ correct point on the psychometric function. The results of $\chi^{2}$-tests indicated that the data were well fit by such regression lines.

Figure 6 shows the slant detection thresholds of two subjects for random-dot patterns as a function of the underlying disparity transformation. Subjects could reliably detect the slant of shear surfaces (sky or ground planes) that had a slant of $1.25 \mathrm{deg}$. In contrast, $2.1 \mathrm{deg}$ of slant was required to detect the slant of expansion-compression surfaces (left or right walls), thus confirming the previously reported anisotropy for perceiving slanting surfaces.

If detection thresholds were governed entirely by the maximum orientation disparity present in these random-dot surfaces, then expansion-compression surface thresholds would be twice as high as shear surface thresholds, since the maximum orientation disparity in expansion-compression surfaces is half that in shear surfaces (see Fig. 3). Detection thresholds for expansion-compression surfaces were, in fact, 1.65 times higher than shear thresholds, which is closer to the result expected on the basis of the average orientation disparity present in the two different surface types $(1.57: 1)$. This result is also comparable to that reported by Rogers and Graham (1983) with threshold and suprathreshold sinusoidally corrugated surfaces.

Figure 7 shows the effect of grid line orientation on surface slant detection thresholds for three subjects. Thresholds for shear surfaces covered with $0 / 90$ deg lines were typically around $1 \mathrm{deg}$ of slant with respect to the 
a
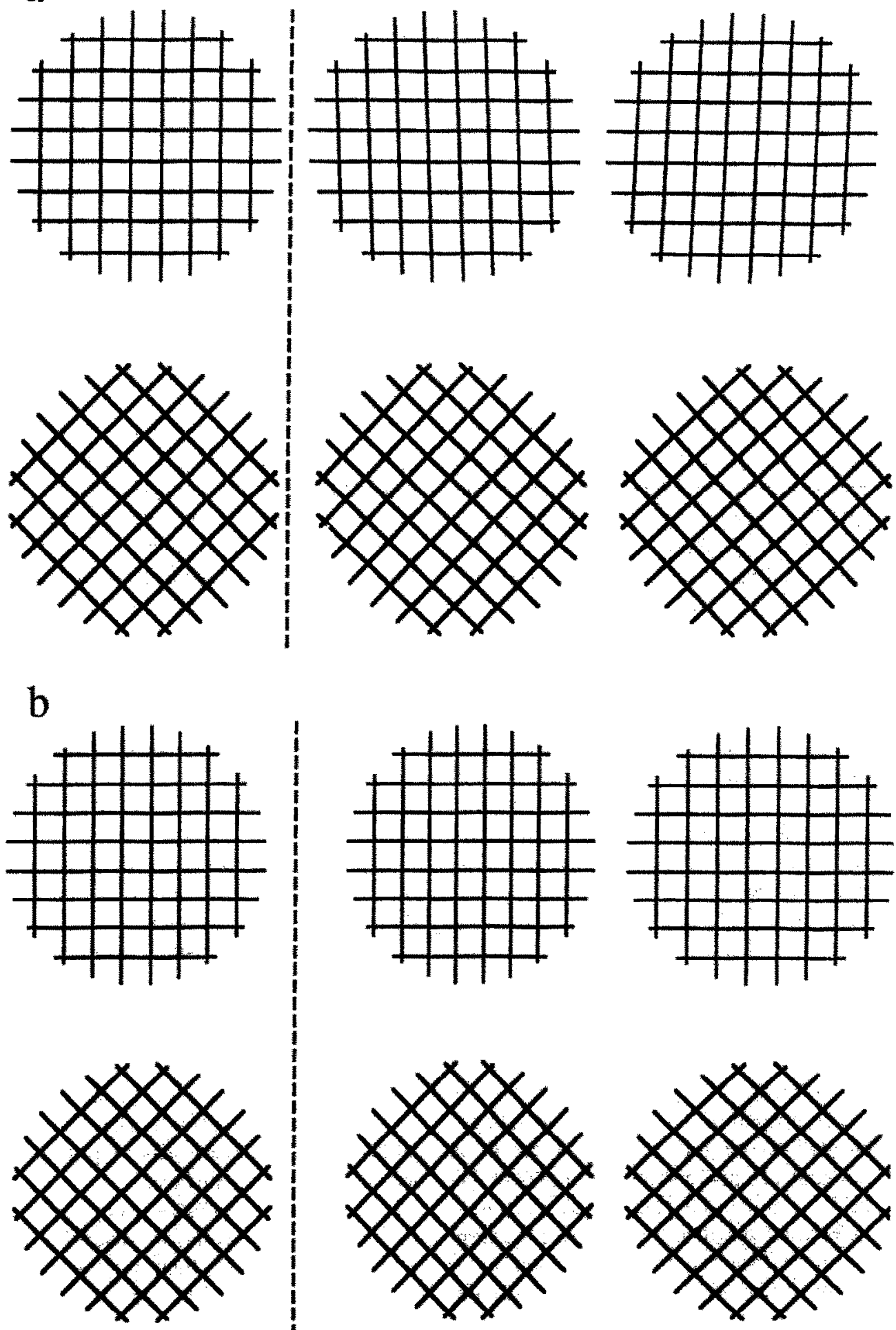

FIGURE 5. Circular grid patches and disparity transformations similar to those used in the experiments. (a) The shearing transformation applied to $0 / 90$ and $\pm 45 \mathrm{deg}$ grids. Top row shows a $0 / 90 \mathrm{deg}$ grid before (to the left of the dashed line), and after the application of an equal and opposite shear transformation. The transformation yields a stereo pair to the right of the dashed line). Botton row is the same for a \pm 45 deg grid. This transformation was used to generate the shear surfaces used in the experiments. (b) The expansion-compression transformation applied to $0 / 90$ and \pm 45 deg grids. This transformation was used to generate the expansion-compression surfaces used in the experiments. This is intended as an illustrative figure, thus the surface slant magnitudes represented here are well above the thresholds we observed, though they should be easy to fuse.

fronto-parallel, and therefore slightly lower than for the same surfaces covered with random dots. In contrast, all three subjects had their highest thresholds when required to detect the slant in an expansion-compression surface covered with $0 / 90$ deg lines. For two observers, as much as $4 \mathrm{deg}$ of slant was required to do the task in this stimulus configuration which generated no orientation disparities. 
(a)



Disparity Transformation

(b)

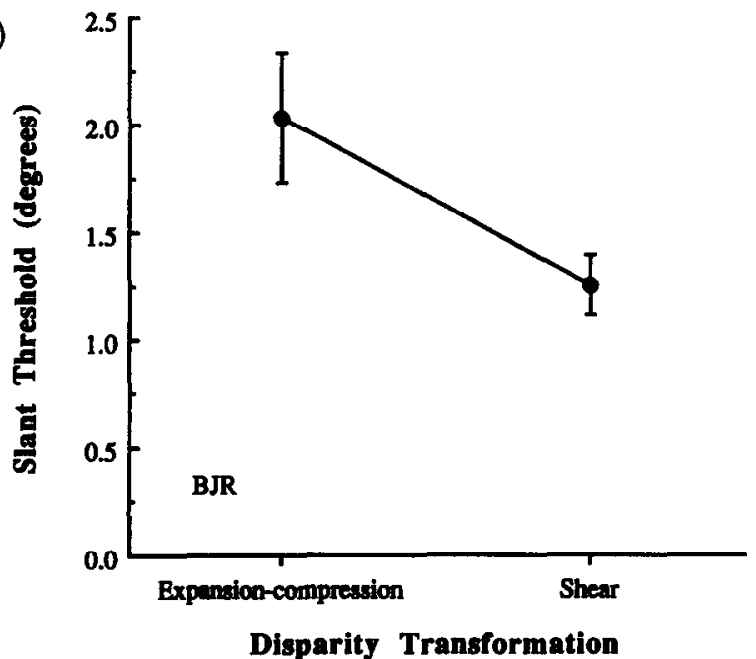

FIGURE 6. Shear and expansion-compression slant detection thresholds of two subjects for random-dot covered stimuli. Minimum detectable surface slant (in degrees from fronto-parallel) is given on the $y$-axis, disparity transformation on the $x$-axis. Error bars are the $95 \%$ confidence intervals.

When the grid line orientation of the expansion-compression surface was changed to $\pm 45 \mathrm{deg}$, thresholds for detection were significantly lower, by a factor of $2.5-3.5$ times, and were comparable to those obtained with the shear surface when it was also covered with $\pm 45 \mathrm{deg}$ lines. Thus, under these conditions, the anisotropy was effectively eliminated when the orientation disparity content of the two surface types was made equal.

These two results strongly suggest that orientation disparity plays an important role in the threshold slant anisotropy. Orientation disparity cannot be the only source of information used, however, because thresholds were not infinitely high in the expansion-compression surface covered with a $0 / 90 \mathrm{deg}$ grid, where there are no orientation disparities between corresponding line elements. In addition to the positional disparities and disparity gradient information present, a weaker orientation disparity cue may have originated from the presence of Fourier energy along the diagonals, or implicit contours (Gillam \& Ryan, 1992) formed by linking up the line intersections.
Looking at the results for the shear surfaces alone, there was a smaller effect $(\sim 1.2-1.4: 1)$ of line orientation on detection thresholds for RBC and SPM, and no effect for observer BJR. On the basis of the maximum

(a)



(b)



(c)

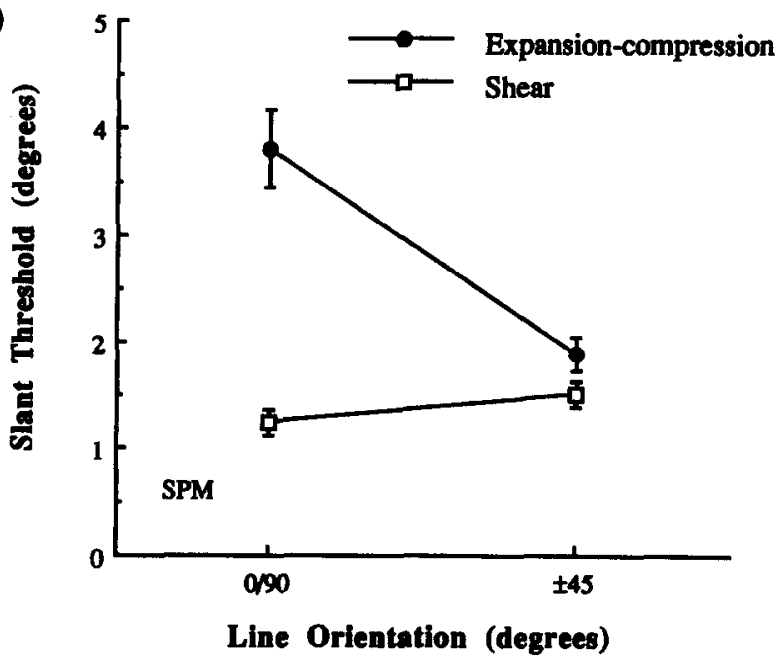

FIGURE 7. Surface slant detection thresholds for shear and expansion-compression surfaces as a function of the orientation of lines on the surface for three subjects. Grid line orientation $(0 / 90$ or $\pm 45 \mathrm{deg})$ is indicated on the $x$-axis, slant detection thresholds (in degrees from fronto-parallel) on the $y$-axis. Shear surfaces are indicated by the open squares, expansion-compression surfaces by the solid circles. Error bars are the $95 \%$ confidence intervals. 
orientation disparity present (Fig. 3), one would predict that shear surfaces covered with $\pm \mathbf{4 5} \mathrm{deg}$ would have detection thresholds twice as high as those for the same surface covered with $0 / 90 \mathrm{deg}$ lines. The average orientation disparity present in these stimuli is the same, however, because the horizontal lines in the shear surface generate zero orientation disparity. Judgments based on the average orientation disparity, therefore, would yield similar thresholds for both grid orientations in this surface configuration. RBC and SPM's results fall in between the two predictions, while BJR's results are close to what would be expected if the average orientation disparity was being extracted.

\section{Discussion}

The major predictions of the orientation disparity hypothesis were borne out in the data. First, the anisotropy in the thresholds for perceiving three-dimensional slant was not in evidence when shear and expansioncompression surfaces were ruled with $\pm 45 \mathrm{deg}$ lines. This is compatible with the use of orientation disparity since, in this case, both shear and expansioncompression surfaces possessed the same magnitude of orientation disparity. Second, expansion-compression surfaces became far harder to see when none of the line elements created any orientation disparities (compare $0 / 90$ deg grid performance with $\pm 45 \mathrm{deg}$ grids or dots). Grid line orientation should not affect thresholds on the basis of positional point disparity processing, because the magnitude of horizontal disparities is the same for corresponding points on a surface of a given slant, regardless of the orientation of surface markings (see Fig. 4). When none of the line elements generated orientation disparities (as with the 0/90 deg expansioncompression surface), thresholds were most probably based on the disparities and/or disparity gradients present but, under these conditions, we found that thresholds were significantly higher.

Several earlier studies of the anisotropy, performed at suprathreshold, obtained results that are also compatible with the use of orientation disparity information. Wallach and Bacon (1976), and Gillam et al. (1984, 1988) found that the latency to perceive depth was longer when stimulus disparities were changing in a horizontal direction (creating expansion-compression patterns of disparity) than when they were changing in a vertical direction (creating shearing patterns of disparity). In Wallach and Bacon's (1976) first experiment, there is a larger component of orientation disparity present in the "transverse" disparity stimulus, which they reported was easier to see, than in their "superpositional" stimulus (their Fig. 2) which is essentially a horizontal expansion of one eye's view, and possesses only a small component of orientation disparity along the oblique. Thus it is possible that the differences in the perceptual latencies were due to the presence of orientation disparity information in the transverse configuration and to its absence in their superpositional stimulus (see also their note 3 , p. 382).
The stimuli of Gillam et al. (1984) which generated the longest latencies and the smallest amounts of perceived depth were horizontal expansions of grids made up of vertical and horizontal dotted lines. These stimuli contained no orientation disparities, but the stimuli which they found to be easiest to perceive did. Gillam et al. (1988) also reported that random-dot stereograms subjected to an expansion compression transformation took much longer to see than those with an underlying shear transformation. The fact that average orientation disparity information present in a shear-defined randomdot stereogram is $57 \%$ larger than that present in a random dot expansion-compression surface as shown earlier, may have contributed to this result. However, in one experiment they doubled the slant present in the surface slanting about a vertical axis (to equate orientation disparity), and still observed longer latencies. making this explanation less likely.

While it may be useful to consider the role of orientation disparity in these earlier studies, other more recent results cast doubt on the sufficiency of an entirely orientation disparity based explanation of suprathreshold slant anisotropies (Mitchison \& McKee, 1990; Gillam \& Ryan, 1992). These studies have shown that the anisotropy persists regardless of line orientation for suprathreshold surfaces. Based on this evidence, it now seems clear that there are important differences in the utilization of orientation disparity in threshold and suprathreshold slant perception.

Because the slant magnitudes of the suprathreshold surfaces used in the studies summarized above were so much greater than in the present study, there may have been factors other than orientation disparity that contributed to the anisotropy, that would not be expected to affect threshold judgments. For example, it has been noted that there may be a differential effect of conflicting perspective information on suprathreshold surfaces slanting about a horizontal or vertical axis, which would not likely operate for threshold slant (Gillam, 1968; Stevens \& Brookes, 1988; Mitchison \& McKee, 1990; Gillam \& Ryan, 1992). This comes about due to common stimulus generation techniques, whereby stereo half-images do not possess any perspective information about the surface slant that is depicted stereoscopically.

Mitchison and McKee (1990) reported that for suprathreshold slanted surfaces, the presence of a square border (i.e. no perspective distortion, thus indicating a fronto-parallel surface) around a surface slanting about a vertical axis lessened the perceived slant of that surface much more than it did for a surface slanting about a horizontal axis. They also found that the anisotropy remained for suprathreshold surfaces, regardless of the superimposed line orientation, although it was reduced when orientation disparities present in the surfaces were the same.

Using surfaces slanting at 15 and $30 \mathrm{deg}$ from the frontoparallel, and of larger angular subtent than Mitchison and McKee (1990), Gillam and Ryan (1992) examined the relative contribution of both conflicting 
perspective and orientation disparity to the anisotropy. They confirmed previous results that perceived slant about a vertical axis was greater for \pm 45 deg grids than for $0 / 90 \mathrm{deg}$ grids, but found that the anisotropy still existed when surfaces were composed of \pm 45 deg grids. They also found, particularly for surfaces slanting about a vertical axis, that the relationship between perceived slant and line orientation was attributable more to conflicting perspective information than to orientation disparity. A stimulus that would normally have a large amount of perspective distortion, such as horizontal lines on a surface slanting about a vertical axis, was perceived as less slanted than a stimulus that would normally possess a smaller amount of perspective distortion, such as oblique or vertical lines on a similarly slanting surface. However, this effect was not found to be significant in surfaces which had a horizontal axis of slant. Furthermore, the results obtained with several stimulus configurations could not be explained by either orientation disparity or conflicting perspective. The results suggest that some form of interaction occurs among orientation disparity, conflicting perspective, and (as yet undefined) configurational factors, with the relative contributions of each depending upon the stimulus composition.

With regard to the present results obtained at threshold, it seems likely that perspective played a much smaller role, if any, because the conflict between the appropriate perspective information and what was presented was negligible for the very small slants used. Thus orientation disparity content would still appear to best describe the anisotropy at slant detection threshold, but for larger slants this information is apparently swamped by conflicting perspective and configurational effects. It would be interesting to determine whether the contribution of orientation disparity to suprathreshold slant perception changes when appropriate perspective information is present.

In addition to their study using suprathreshold surface slants, Mitchison and McKee (1990) measured slant detection thresholds to test our orientation disparity hypothesis, which first appeared in Cagenello and Rogers (1988a). Consistent with our results, they found lower detection thresholds for five out of six subjects who obtained measurable thresholds, for expansion-compression surfaces with $\pm 45 \mathrm{deg}$ grids compared to those containing $0 / 90 \mathrm{deg}$ grids. This result is clearly consistent with the use of orientation disparities.

Contrary to our results, however, they did not observe equal thresholds for shear and expansion-compression surfaces ruled with \pm 45 deg lines. Subject SPM, for example, required 65 times more disparity to see slant about a vertical axis (expansion-compression disparity patterns), than slant about a horizontal axis (shear disparity patterns), when the surfaces were covered with \pm 45 deg lines. Moreover, four of their ten subjects could not detect slant in expansion-compression surfaces at all, regardless of the line orientation used. On average, the thresholds they obtained (when expressed as dis- parity gradients) were approximately 15 times higher than ours for both shear and expansion compression surfaces.

The principal reason for these discrepancies, we feel, lies in the difference in stimulus size used in the two studies. The surface patches in their threshold study subtended only $0.75 \mathrm{deg}$ of visual angle, and were composed of five lines separated from each other by $11 \mathrm{~min}$ arc. Our surface patches, on the other hand, subtended $10.66 \mathrm{deg}$, and were composed of eight lines separated by $1.3 \mathrm{deg}$. In limiting the stimulus size to foveal dimensions, it is possible that Mitchison and McKee were handicapping the use of orientation disparity, which necessarily depends on extended spatial features to be processed accurately.

In support of this notion, there is evidence that for line stimuli subtending $<2 \mathrm{deg}$ of visual angle, position rather than orientation cues are used in line orientation discrimination tasks (Orban, Vandenbussche \& Vogels, 1984). Therefore, it may have been difficult for subjects in the Mitchison and McKee study to use the limited amount of orientation information present in their stimuli, so that observers were obliged to use positional disparity cues.

The clearest evidence that stimulus size is responsible for the differences in the results of the two studies is that one of the authors of Mitchison and McKee (1990) (SPM, Fig. 7) obtained similar results to our own when presented with the $10.66 \mathrm{deg}$ stimuli in our set-up. The question of why the anisotropy exists for small stimuli remains unanswered, of course -if it is because orientation disparity information is ineffective, then it is unclear why the remaining information present in small surface patches is subject to a processing asymmetry in the visual system.

\section{EXPERIMENT TWO}

To better understand the interaction between stimulus size and slant detection thresholds, a second experiment was undertaken in which detection thresholds were measured over a large range of sizes of the surface patches. Manipulating the size of a slanted surface patch would be expected to have a significant effect on detection thresholds because the size of the patch determines the maximum disparity (between the outer edges of the patch) that is present in the stimulus. On the other hand, patch size would be expected to have rather little effect on detection thresholds (within limits) if either orientation disparity or disparity gradient was the main source of information used to perform the task, because each remains constant for a given surface slant of any visible size. Therefore, the extent to which detection thresholds vary as a function of stimulus size could provide information as to whether different strategies (i.e. using point disparities, disparity gradients, or orientation disparities) underlie task performance for different stimulus sizes.

Specifically, doubling the visible size of a slanted surface doubles the maximum disparity present (at 
the edges). Thus if a constant magnitude of disparity is required for slant detection, thresholds would halve when stimulus size doubles. On the other hand, thresholds would be expected to remain constant with changes in stimulus extent, if disparity gradient or orientation disparity information underlies threshold performance, since these sources of information remain constant with varying stimulus size. This technique was used previously by Braddick (1968; cited by Braddick, 1979), who manipulated stimulus size to show that the interocular difference in orientation, rather than the maximum positional disparity in a display, determines whether or not diplopia occurs.

\section{Methods}

Stereoscopic slant thresholds were measured for shear surfaces covered with lines of \pm 45 or $0 / 90 \mathrm{deg}$. The same apparatus and procedure as the previous experiment were used. Stimulus size was varied by blanking the displays to all but a circular region of the stimulus with a diameter of $1.33,2.66,5.33,10.66$, or $21.33 \mathrm{deg}$ of visual angle. Two subjects took part, each making a total of 280 observations ( 40 per point of the psychometric function) at each stimulus size for each grid orientation.

\section{Results}

Thresholds were again based on the reciprocal of the slope of the best-fitting regression line using probit analysis. Figure 8 shows the disparity difference between the outer edges of the surface patch required for detection as a function of stimulus size for the two subjects. It can be seen that disparity thresholds increased with increasing patch size. For the smallest stimulus sizes, however, disparity increased by a much smaller amount than at the largest sizes; it doubled for larger sizes, which would be expected if disparity gradient or orientation disparity remained constant, but increased by only 1.4 times on average at the two smallest stimulus sizes. This pattern of results is consistent with there being a larger contribution of positional disparity to threshold performance for stimuli subtending 1.3 and $2.6 \mathrm{deg}$ than for the larger stimulus sizes.

To isolate the way in which slant thresholds vary with stimulus size, the data from Fig. 8 are re-plotted in Fig. 9 as slant thresholds. In this case, thresholds remained approximately constant at close to $1 \mathrm{deg}$ of slant for stimulus sizes of $5.33 \mathrm{deg}$ and above, even though the maximum disparity present in the display doubled for each doubling of stimulus size. Thresholds increased to 2-3 deg for the smallest stimulus, representing a 2-3:1 overall difference in thresholds over the 16:1 range of stimulus sizes used. The greater influence of positional disparity (as mentioned above, Fig. 8) is reflected in this graph in the region where thresholds are not constant, at the smallest simulus sizes. Grid line orientation had some effect at the smallest stimulus sizes, but virtually none at the larger stimulus sizes where the data curves have zero slope. Thresholds in this region are therefore consistent with the extraction by the visual (a)

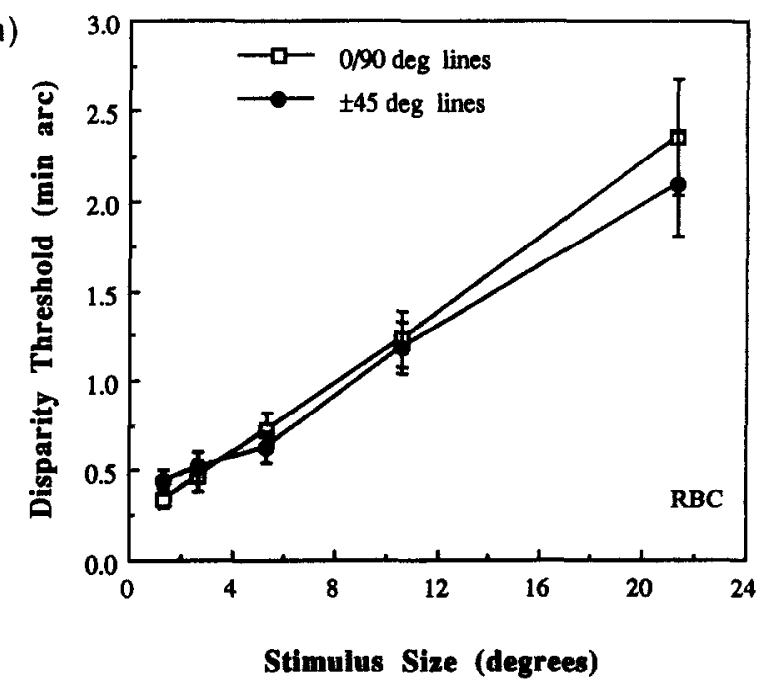

(b)



FIGURE 8. Disparity required to detect surface slant as a function of stimulus size for two observers. Plot symbols indicate grid line orientation, $0 / 90$ or $\pm 45 \mathrm{deg}$. Stimulus size $(1.33,2.66,5.33,10.66$, $21.33 \mathrm{deg}$ ) is indicated on the $x$-axis, disparity at threshold (min arc) on the $y$-axis. Error bars are the $95 \%$ confidence intervals.

system of either average orientation disparity, or disparity gradient.

\section{Discussion}

As was found for line orientation discrimination (Orban et al., 1984), positional information plays a larger role in the detection of stereoscopic slant for patches subtending $\sim 2$ deg or less than in larger stimuli. This could explain why Mitchison and McKee (1990) did not find as strong an influence of orientation disparity on the anisotropy as reported here, because the positional disparities of their small stimuli were the more salient slant cue. Stimulus size can also explain the differences in size of thresholds between our results and those of Mitchison and McKee (1990). Our results show that thresholds were considerably larger with the smaller patch sizes, although they never reached the values that Mitchison and McKee reported. However, given that our smallest stimulus in this experiment $(1.33 \mathrm{deg})$ was still almost twice the size of their stimuli, it is not 
(a)



(b)

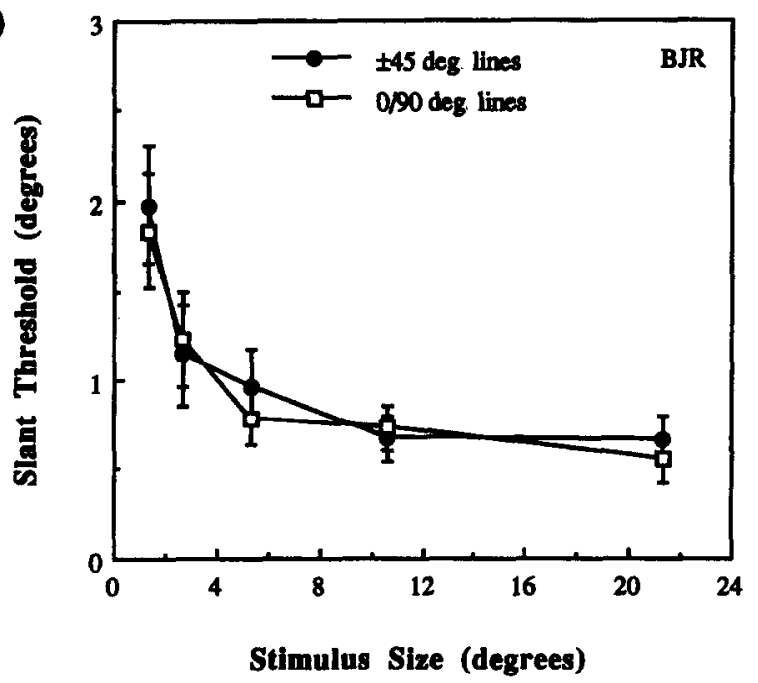

FIGURE 9. Surface slant detection thresholds as a function of stimulus size for two observers. Plot symbols indicate grid line orientation, $0 / 90$ or $\pm 45 \mathrm{deg}$. Stimulus size $(1.33,2.66,5.33,10.66$, $21.33 \mathrm{deg}$ ) is indicated on the $x$-axis, surface slant at threshold (degrees from fronto-parallel) on the $y$-axis. These are the same data as in Fig. 8, re-plotted as slant thresholds. Error bars are the 95\% confidence intervals.

surprising that our thresholds were significantly lower than theirs.

The constancy of slant detection thresholds for patches subtending $5.33 \mathrm{deg}$ and above (Fig. 9) is certainly consistent with the use of orientation disparities or the disparity gradient present.

\section{GENERAL DISCUSSION}

The principal finding of the experiments reported here is that the magnitude of the perceptual anisotropy in surface slant detection is affected by the orientation of lines covering stereoscopic surfaces. The most plausible interpretation of these results is that orientation disparity provided the information used by subjects to perform the task. That the visual system is apparently able to use this information is of interest, given the well-established theoretical importance of binocular differences in orientation information.

Koenderink and van Doorn (1976) laid the theoretical groundwork for the analysis of binocular image orientation information, showing that the deformation component of the disparity field specifies the disparity gradient, and that it can be measured (approximately) by monitoring the difference in angles formed by corresponding pairs of intersecting lines. This quantity, an angular disparity, has the important property that it remains invariant with whole-field image rotations, as would occur when the eyes are misaligned about their lines of sight (cyclovergence). However, recent studies have apparently provided evidence against the use of the deformation component of the disparity field. Cagenello (1990), and Gillam and Rogers (1991) found that the perceived slant of surfaces defined by horizontal and vertical shear, pure curl, and pure deformation was not dependent on the deformation component of the underlying disparity fields. Instead, perceived slant was found to depend on the other slant information present including disparities, disparity gradients, and orientation disparities.

Orientation disparity, as it is commonly defined and used in this paper, also varies with surface slant, but differs from the angular disparity approximation to deformation, in the following ways. First, a single binocularly corresponding line is required to derive orientation disparity, unlike angular disparity which requires a pair of lines at different absolute orientations in each image (Koenderink, 1986). As a result, orientation disparity varies with the state of torsional alignment between the two eyes. Cagenello and Rogers (1990) have reported that torsional misalignment affects perceived slant. Second, orientation disparities must be scaled by the orientation of lines on three-dimensional surfaces to obtain an estimate of slant, whereas angular disparity is directly related to surface slant. This is a consequence of the geometry which is expressed in Fig. 3; orientation disparity for a fixed surface slant depends on absolute line orientation. The perspective effect described by Gillam and Ryan (1992) would, in principle, require a similar scaling process and it would be interesting to see if the influence of contradictory or appropriate perspective information on perceived slant varies with the orientation of lines in the stimulus.

Wildes $(1989,1991)$ showed that pairs of orientation disparities can be used to calculate an angular disparity, which avoids the need for scaling. This is essentially equivalent to the approximation to deformation suggested by Koenderink and van Doorn (1976). A computer algorithm that Wildes devised using this technique was able to recover robust estimates of surface slant from stereo images of natural scenes. Jones and Malik (1992) have also successfully made use of orientation disparity information in their computer program, which recovers surface shape characteristics from natural stereo images.

Several researchers have argued for the use of orientation disparity by the visual system (von der Heydt, 1978; 
Ninio, 1985; DeValois et al., 1975; Gillam \& Rogers, 1991). von der Heydt (1978; see also von der Heydt et al., 1981) presented subjects with uncorrelated dynamic noise grating stereograms which contained varying magnitudes of orientation disparity. Although there were no consistent positional disparities from frame to frame, surface slant was perceived for a large range of orientation disparities, providing evidence for a distinct neural mechanism for orientation disparity processing. While this illustrates that orientation disparity can be processed in the absence of all other consistent slant information, it does not indicate how such a mechanism would function in normal circumstances, when other information about surface slant is available.

Ninio (1985) manipulated corresponding line pairs in line stereograms so that they contained differing amounts of orientation and positional disparity. He found that line pairs with an orientation disparity component were more likely to appear as smoothly slanting than those without. However, orientation disparity was not manipulated independently of positional disparity and disparity gradient, because random-line orientations were used. Thus it is not clear that the results are best explained by orientation disparity processing.

DeValois et al. (1975; see also DeValois \& DeValois, 1988 ) reported a slant aftereffect in depth which generalized to test surfaces that had different absolute disparities from those in the adapting surface (by introducing a disparity offset between test and adapting surfaces), thus ruling out disparity adaptation. They argued that orientation disparity adaptation could explain the results, though adaptation of a disparity gradient mechanism could also have been a factor. These two possibilities could be teased apart by using stimuli similar to those we used in the present study, because they provide independent control of orientation disparity and disparity gradient.

Gillam and Rogers (1991) (whose results were summarized above) concluded that orientation disparity was the stimulus feature that best accounted for their findings. The results they obtained, however, would also be predicted by the positional disparities and disparity gradients present in their stimuli.

The inconclusive nature of three of the studies summarized above regarding the role of orientation disparity is perhaps due to the co-variance of positional disparity, disparity gradient, and orientation disparity in normal stereoscopic stimuli depicting slanting surfaces. The studies that independently manipulated orientation disparity (the present study and von der Heydt, 1978) were able to determine that orientation disparity can indeed play a role in slant perception. In addition, the presence of equal magnitudes of orientation disparity in shear and expansion-compression surfaces in the present study eliminated the anisotropy in slant detection, suggesting the importance of orientation disparity.

Earlier explanations of the anisotropy have typically been rc-descriptions of the phenomenon in terms of the stimulus properties or the viewing geometry. For example, the fact that the anisotropy is consistently related to the form of the underlying disparity transformation led to a suggestion of an asymmetry in the visual system's processing of disparities which change in a vertical direction (orthogonal to the axis joining the two eyes) vs a horizontal direction (parallel to the axis joining the two eyes). That is, it was hypothesized that the visual system is insensitive to disparity changes in a horizontal direction, and that disparity changes in a vertical direction are more effective (Wallach \& Bacon, 1976; Rogers \& Graham, 1983; Gillam et al., 1984; Tyler, 1991).

More recently, Mitchison and Westheimer (1990), and Mitchison and McKee (1990) have proposed an explanation of the anisotropy in terms of the stereo viewing geometry. Eccentric binocular viewing produces whole-field gradients of disparity which are larger in a horizontal direction than in a vertical direction. Eccentric fixation would therefore cause spurious horizontal gradients of disparity, making that information an unreliable indicator of surface slant. For this reason they hypothesized that there is no in-built reference for surfaces slanting about a vertical axis, which makes them harder to see. However, curved surfaces also generate an anisotropy (Rogers \& Graham, 1983; Cagenello \& Rogers, 1988b; Rogers \& Cagenello, 1989), whereas the detectability should be unaffected by the introduction of a whole-field horizontal disparity gradient, because the local changes in disparity that define the curvature (the second derivative of positional disparity) remain unaltered.

Neither of these earlier accounts of the anisotropy can be easily interpreted in terms of the underlying mechanisms. In contrast, if orientation disparity provides an adequate explanation of the anisotropy at the thresholds of slant perception, then it is not difficult to imagine a mechanism that would be capable of extracting binocular differences in the orientation of line elements (Blakemore et al., 1972; Nelson et al., 1977; Hanny, von der Heydt \& Poggio, 1977).

Our analysis of the geometry of orientation disparity shows that the magnitude and direction of the threshold slant anisotropy is consistent with the magnitude of orientation disparity in stereoscopic projections of slanted surfaces. In addition, we have psychophysical evidence that the anisotropy can be made larger or smaller by varying the absolute orientation of lines covering stereoscopic surfaces, which alters the orientation disparity present but not positional disparities or disparity gradients. These results therefore provide further evidence for the use of orientation disparities by the visual system, and provide the basis of an explanation of the anisotropy in stereoscopic slant detection.

\section{RFFERENCES}

Bishop, P. O. (1978). Orientation and position disparities in stereopsis In Cool, S. J. \& Smith, E. L. (Eds), Frontiers in visual science (pp. 336-350). New York: Springer. 
Blakemore, C. B., Fiorentini, A. \& Maffei, L. (1972). A second neural mechanism of binocular depth discrimination. Journal of Physiology, 226, 725-749.

Braddick. O. J. (1968). Binocular fusion and perceptual analysis. Ph.D. dissertation, Cambridge University, Cambridge.

Braddick, O. J. (1979). Binocular single vision and perceptual processing. Proceedings of the Royal Society of London B, 204, 503-512.

Cagenello, R. (1990). Perception and representation of stereoscopic slant and curvature. Ph.D. dissertation, Oxford University, Oxford.

Cagenello, R. B. \& Rogers, B. J. (1988a). Local orientation differences affect the perceived slant of stereoscopic surfaces. Investigative Ophthalmology and Visual Science (Suppl.), 29, 399.

Cagenello, R. B. \& Rogers, B. J. (1988b). The perception of curved stereoscopic surfaces: The role of local differences in image curvature. Perception, 17, 366.

Cagenello, R. B. \& Rogers, B. J. (1990). Orientation disparity, cyclotorsion, and the perception of surface slant. Investigative Ophthalmology and Visual Science (Suppl.), 31, 97.

DeValois, R. L. \& DeValois, K. K. (1988). Spatial vision. New York: Oxford University Press.

DeValois, K. K., von der Heydt, R., Adorjani, C. S. \& DeValois, R. L. (1975). A tilt aftereffect in depth. Investigative Ophthalmology and Visual Science (Suppl.), 15, 90.

Finney, D. J. (1971). Probit analysis. Cambridge: Cambridge University Press.

Gillam, B. (1968). The perception of slant when stereopsis and perspective conflict: Experiments with aniseikonic lenses. Journal of Experimental Psychology, 72, 559-564.

Gillam, B. \& Rogers, B. (1991). Orientation disparity, deformation and stereoscopic slant perception. Perception, 20, 441-448.

Gillam, B. \& Ryan, R. (1992). Perspective, orientation disparity, and anisotrophy in stereoscopic slant perception. Perception, 21, 427-439.

Gillam, B., Chambers, D. \& Russo, T. (1988). Postfusional latency in stereoscopic slant perception and the primitives of stereopsis. Journal of Experimental Psychology: Human Perception and Performance, 14, 163-175.

Gillam, B. J., Flagg, T. \& Finlay, D. (1984). Evidence for disparity change as the primary stimulus for stereoscopic processing. Perception \& Psychophysics, 36, 559-564.

Hanny, P., von der Heydt, R. \& Poggio, G. F. (1977). Binocular neuron responses to tilt in depth in the monkey visual cortex: Evidence for orientation disparity processing. Union of Swiss Societies of Experimental Biology Abstracts, A26.

von der Heydt, R. (1978). Stereoscopic perception of orientation disparity. Investigative Ophthalmology and Visual Science (Suppl.), 19, 263.

von der Heydt, R., Hanny, P. \& Dursteler, M. R. (1981). The role of orientation disparity in stereoscopic perception and the development of binocular correspondence. In Grastyan, E. \& Molnar, P.
(Eds), Advances in Physiological Sciences, 16 (pp. 461-470). New York: Pergamon Press.

Jones, D. G. \& Malik, J. (1992). Determining three-dimensional shape from orientation and spatial frequency disparities. Proceedings of the European Conference on Computer Vision, Genoa (pp. 661-669).

Koenderink, J. J. (1986). Optic flow. Vision Research, 26, 161-180.

Koenderink, J. J. \& van Doorn, A. J. (1976). Geometry of binocular vision and a model for stereopsis. Biological Cybernetics, 21, 29-35.

Mitchell, D. E. \& O'Hagan, S. (1972). Accuracy of steresscopic localization of small line segments that differ in size or orientation for the two eyes. Vision Research, 12, 437-454.

Mitchison, G. J. \& McKee, S. P. (1990). Mechanisms underlying the anisotropy of stereoscopic tilt perception. Vision Research, 30, 1781-1791.

Mitchison, G. J. \& Westheimer, G. (1990). Viewing geometry and gradients of horizontal disparity. In Festschrift for H. B. Barlow. Cambridge: Cambridge University Press.

Nelson, J. I., Kato, H. \& Bishop P. O. (1977). Discrimination of orientation and position disparities by binocularly activated neurons in cat striate cortex. Journal of Neurophysiology, 40 , 260-283.

Ninio, J. (1985). Orientational versus horizontal disparity in the stereoscopic appreciation of slant. Perception, 14, 305-314.

Orban, G. A., Vandenbussche, E. \& Vogels, R. (1984). Human orientation discrimination tested with long stimuli. Vision Research, $24,121-128$

Rogers, B. J. \& Cagenello, R. B. (1989). Disparity curvature and the perception of three-dimensional surfaces. Nature, 339, 135-137.

Rogers, B. \& Graham, M. (1982). Similarities between motion parallax and stereopsis in human depth perception. Vision Research, 22 $261-270$

Rogers, B. J. \& Graham, M. E. (1983). Anisotropies in the perception of three-dimensional surfaces. Science, 221, 1409-1411.

Stevens, K. A. \& Brookes, A. (1988). Integrating stereopsis with monocular interpretations of planar surfaces. Vision Research, 28 , 371-386.

Tyler, C. W. (1991). Cyclopean vision. In Cronly-Dillon, J. R. (Ed.), Vision and visual dysfunction. Vol. 9: Binocular vision (pp. 38-74) Boca Raton, Fla: CRC Press

Wallach, H. \& Bacon, J. (1976). Two forms of retinal disparity. Perception \& Psychophysics, 19, 375-382.

Wildes, R. P. (1989). Surface orientation from binocular stereo orientational disparity. SPIE Intelligent Robots and Computer Vision VIII (pp. 309-317)

Wildes, R. P. (1991). Direct recovery of three-dimensional scene geometry from binocular stereo disparity. IEEE Transactions on Pattern Analysis and Machine Intelligence, 13, 761-774.

Acknowledgements-We thank Aries Arditi and Elizabeth Johnston for their helpful comments on earlier versions of this paper. 